\title{
CMEARTICLE
}

\section{Management of sepsis in acute care}

Kay Choong $\underline{\mathrm{See}}^{1}$, MRCP, MPH

A 40-year-old woman with diabetes mellitus and hypertension visited your clinic for routine follow-up. She appeared lethargic and had a history of worsening fever and dysuria over the past three days. Her vital signs were as follows: respiratory rate 22 breaths/min, heart rate 120 beats/min, blood pressure $100 / 60 \mathrm{mmHg}$. On examination, she had normal mental status, normal chest findings, left renal angle tenderness and a capillary refill time of four seconds. Urine dipstick testing showed leukocytes $3+$ and nitrites $1+$, indicating the presence of a urinary tract infection. You suspected sepsis due to left pyelonephritis.

\section{DIAGNOSIS AND DEFINITION OF SEPSIS}

Sepsis is a dysregulated host response to infection, which is a disease caused by pathogens (viruses, bacteria, fungi and parasites). The key to identification of sepsis is suspicion of infection, which requires clinical experience and skill. Overt symptoms and signs of infection include fever and site-specific inflammation (i.e. cellulitis, inflamed joints, purulent sputum). Subtle symptoms and signs of infection are more common in immunocompromised patients and older individuals, who are not able to mount robust inflammatory responses to pathogens and may remain afebrile. ${ }^{(1)}$ These subtle symptoms include lethargy, mild confusion and mild behavioural changes.

Sometimes, infection can be confirmed immediately through physical examination (e.g. severe cellulitis) or via point-of-care testing (e.g. urine dipstick analysis). At other times, the infection is detected after a delay and only confirmed using cultures from otherwise sterile sites, such as blood, cerebrospinal fluid and joint fluid. Regardless of the time of confirmation, when infection is suspected, action should be taken to evaluate its severity and to provide empirical treatment as appropriate.

The severity of infection can be determined from the host's response. In milder cases, the infected person's vital signs remain normal. In more severe cases, which we term sepsis, the infected person will demonstrate a dysregulated host response, marked by any of the following features: hypoxaemic respiratory failure, hypotension, hypoperfusion, depressed mentation, raised bilirubin, thrombocytopaenia or raised creatinine (indicating acute kidney injury). Septic shock is the most severe form of infection and is diagnosed when severe vasodilatation overwhelms cardiac compensation, leading to hypotension despite adequate volume resuscitation.

Blood pressure can be measured in a non-invasive or invasive manner; the mean arterial pressure is considered as the sum of one-third of the systolic blood pressure and two-thirds of the diastolic blood pressure. Hypotension is defined as a mean arterial pressure $<65 \mathrm{mmHg}$. ${ }^{(2)}$ Vasopressor-dependent hypotension means that the patient needs a vasoconstrictive agent (e.g. intravenous infusion of noradrenaline, vasopressin or dopamine) to maintain a mean arterial pressure of at least $65 \mathrm{mmHg}$.

Hypoperfusion can exist with or without hypotension. It is marked by an increased capillary refill time $>3$ seconds or an increased blood lactate level $>2 \mathrm{mmol} / \mathrm{L}$ (measured with arterial, venous or capillary blood). As septic shock strictly requires the combined presence of vasopressor-dependent hypotension and a blood lactate level $>2 \mathrm{mmol} / \mathrm{L}$, despite adequate volume resuscitation, it is unlikely to be diagnosed by frontline clinicians in the pre-hospital and general ward settings.

In clinical settings where laboratory testing is unavailable, sepsis can be suspected but not confirmed when at least two of the following three criteria are present (i.e. quick Sequential Organ Failure Assessment [qSOFA] score $\geq 2)^{(3)}$ : (a) altered mental status (i.e. Glasgow Coma Scale score < 15); (b) respiratory rate $\geq 22$ breaths/min; and (c) systolic blood pressure $\leq 100 \mathrm{mmHg}$. Given that this score is more specific than sensitive for sepsis, sepsis remains possible if the patient appears ill to the clinician or has hypoperfusion, even if the qSOFA score is $<2$.

\section{APPROACH TO SEPSIS FOR THE FRONTLINE CLINICIAN}

Sepsis is prevalent and contributes to a large burden of morbidity and mortality worldwide. ${ }^{(4)}$ Hence, expeditious treatment is paramount to minimise the morbidity and mortality. For instance, every hour of delayed antibiotic administration increases the risk of death from septic shock by $7.6 \% .{ }^{(5)}$ To avoid treatment delays, possible sepsis should be regarded as seriously as definite sepsis.

Patients with community-acquired sepsis often present in the pre-hospital setting, while patients with hospital-acquired sepsis often present in the general wards. Early recognition of sepsis is the critical first step towards improving care quality and outcomes. Frontline clinicians in the clinic, emergency department and general wards need to be prepared to identify possible sepsis using simple bedside tools such as qSOFA $^{(3)}$ as well as capillary

${ }^{1}$ Division of Respiratory and Critical Care Medicine, Department of Medicine, National University Hospital, Singapore 
refill time. ${ }^{(6)}$ Once sepsis is identified, frontline clinicians must be poised to initiate simple but life-saving therapy (i.e. timely antibiotics and fluid challenge).

\section{Key clinical concept 1: tissue perfusion}

Tissue hypoperfusion occurs owing to mismatched oxygen supply (relatively low) and demand (relatively high), or through the inability of end-organs to utilise delivered oxygen, such as from cytotoxic damage or microcirculatory dysfunction. Measurement of blood pressure or cardiac output provides information about circulatory supply but not about demand or tissue hypoperfusion. Common methods of detecting hypoperfusion involve bedside examination methods (e.g. capillary refill time, skin mottling) and blood lactate measurements.

For the frontline clinician, capillary refill time is of particular value given its ready availability, low cost and repeatability. It can be measured in any digit, although it can be impossible to measure in the presence of severe peripheral vascular disease. Conversely, skin mottling is a late sign of tissue hypoperfusion, while blood lactate can be elevated without tissue hypoperfusion (e.g. beta-agonist use, liver dysfunction, metformin use).

Capillary refill time can be measured in a standardised and repeatable manner (Box 1). A capillary refill time exceeding three seconds indicates the presence of hypoperfusion ${ }^{(6)}$ and requires treatment. Possible differentials for a prolonged capillary refill time include severe peripheral vascular disease and cold peripheries, although these conditions can be readily recognised and the latter can be mitigated by warming the digits.

\section{Key clinical concept 2: timely antibiotics and source control}

Antimicrobial therapy for sepsis needs to be delivered quickly, ${ }^{(7)}$ within three hours of presentation and preferably within one hour. (2) Given that the symptoms and signs of sepsis may be non-specific, overtreatment with antimicrobials is possible, generating concerns about excessive adverse drug reactions and antimicrobial resistance. To mitigate overtreatment and antimicrobial resistance, prompt de-escalation or cessation of antimicrobials is important once clinical progression is clear and culture results are available 2-3 days later. In addition, the duration of antibiotics needs to be minimised. Such a strategy avoids placing undue responsibility for appropriate antimicrobial use on the frontline clinician, and minimises the risk and complications of delayed treatment of ill patients. If available, rapid molecular techniques may allow earlier adjustments to the antimicrobial regime. ${ }^{(8)}$ These techniques may also help to elucidate the aetiological agent for culture-negative sepsis, which has a mortality rate similar to that of culture-positive sepsis. ${ }^{(9)}$

Empiric antimicrobial therapy should be tailored to cover the responsible pathogens, which are commonly bacteria or viruses. Under conditions of clinical uncertainty, epidemiological evidence supports broad-spectrum coverage of Gram-positive and Gram-negative bacteria. ${ }^{(10)}$ If appropriate and available, antivirals (e.g. against influenza or severe acute respiratory syndrome coronavirus 2) can be considered.(2) Overall, the

\section{Box 1. Measurement of capillary refill time:}

- Select the right or left index finger. If not available, choose another finger or toe of similar size.

- Apply firm pressure to the ventral surface of the distal phalanx with your thumb.

- Increase the pressure until the nailbed of your thumb starts to blanch, and then maintain pressure for 10 seconds (mentally count 'one-a-thousand', 'two-a-thousand' ... 'ten-a-thousand').

- Release your thumb and count the number of seconds required for return of normal skin colour (i.e. the capillary refill time).

- A capillary refill time $>3$ seconds is abnormal.

suspected source of infection, local epidemiological data and travel history are important guides for antimicrobial choice. In our case, the patient's urinary tract infection should be treated with antimicrobials that target the genitourinary bacteria, particularly Escherichia and Klebsiella species. ${ }^{(11)}$

To ensure adequate systemic levels of antimicrobials, the route of administration of antimicrobials is often intravenous. Nonetheless, oral administration of antimicrobials with good bioavailability (e.g. oral fluoroquinolones) is a reasonable alternative. In the presence of renal impairment, dose adjustments may be unnecessary for the first few days of treatment, given the augmented renal clearance in sepsis. ${ }^{(12)}$ Otherwise, therapeutic drug monitoring may be available to guide dosing of some antimicrobials such as vancomycin and aminoglycosides.

Besides antimicrobial therapy, some forms of sepsis necessitate urgent source control to effect cure and minimise mortality. ${ }^{(13)}$ Empyema requires chest tube drainage, cholangitis requires endoscopic or percutaneous biliary drainage, and necrotising fasciitis requires tissue debridement. Severely ill patients who require surgery may need to be stabilised via endotracheal intubation and mechanical ventilation. However, waiting for further stabilisation of vital parameters using medications alone may ironically result in delayed treatment and poor clinical outcomes. As such, surgery for source control in septic patients should not be delayed if hypoperfusion and hypotension cannot be fully reversed using medications alone.

\section{Key clinical concept 3: fluid challenge}

Sepsis-related haemodynamic decompensation is complex, with multiple possible causes including hypovolaemia, vasodilatation and septic cardiomyopathy. Besides treating the underlying infection, direct reversal of these pathological changes involves fluid repletion for hypovolaemia, vasoconstrictive agents (vasopressors) for vasodilatation and inotropic agents for septic cardiomyopathy.

For any septic patient with hypoperfusion, determining the cause is not straightforward; hypovolaemia, vasodilatation and septic cardiomyopathy may be present individually or in combination. Furthermore, precise measurements for total body water and systemic vascular resistance may not be available, even in the hospital setting. Given the ready availability of intravenous fluids, when sepsis-related hypoperfusion exists, hypovolaemia is often assumed to be present and is empirically treated. Should a patient benefit from fluid therapy, the cardiac stroke volume should increase by at least $10 \%$. $^{(14,15)}$ 


\section{Box 2. Performing a fluid challenge:}

- Insert as large an intravenous cannula as possible, preferably 20 gauge or larger

- Prepare $500 \mathrm{~mL}$ of intravenous crystalloid (e.g. normal saline, lactated Ringer's/Hartmann's solution)

- Decide on the patient's body position and keep it constant
(a) Supine or
(b) Head up $30^{\circ}-45^{\circ}$ or
(c) Trendelenburg

- Attach blood pressure cuff on one limb (arm preferred); keep the selected limb constant
(a) Right arm or
(b) Left arm or
(c) Right leg or
(d) Left leg

- Record the initial parameters immediately before fluid infusion

(a) Systolic blood pressure $(\mathrm{mmHg})$

(b) Diastolic blood pressure $(\mathrm{mmHg})$

- Run the fluid challenge within 30 minutes and prepare to return at the end of the infusion

- Record the final parameters immediately on completion of fluid infusion

(a) Systolic blood pressure $(\mathrm{mmHg})$

(b) Diastolic blood pressure $(\mathrm{mmHg})$

- Check capillary refill time. If this has normalised (i.e. 3 s or less), consider stopping further fluid loading

Fluid overload, however, can lead to pulmonary oedema, raised intra-abdominal pressure, tissue oedema, and eventually, increased mortality. ${ }^{(2)}$ As such, intravenous fluid administration needs to be performed via carefully monitored fluid challenges, using pulse pressure (i.e. the difference between systolic and diastolic blood pressure) as a clinically convenient parameter in lieu of cardiac stroke volume. ${ }^{(15)}$ The initial choice of fluid can be either normal saline or a balanced crystalloid (e.g. lactated Ringer's or Hartmann's solution), ${ }^{(16)}$ but the performance of a fluid challenge needs to be standardised (Box 2). In particular, a sufficiently large bolus of fluid needs to be administered intravenously and rapidly, and the blood pressure should be measured within 1-2 minutes after the end of the fluid bolus. ${ }^{14,17}$ If the pulse pressure increases by at least $10 \%$, the fluid challenge should be repeated. If the pulse pressure does not increase by at least $10 \%$, further fluid loading will not benefit the patient. Finally, if hypoperfusion resolves, consider ceasing further fluid loading.

\section{FOLLOW-UP CARE}

Practically, if a septic patient presents in the outpatient setting, immediate assessment, treatment and escalation to the emergency department are recommended. When such patients are seen in the emergency department or in the general ward, those who require mechanical (invasive or non-invasive) ventilation or vasopressors should be transferred to an intensive care unit (ICU) unless they have a care limitation order. Such transfers should also be expedited, as excessive emergency department boarding times and delayed intensive care unit transfers of critically ill patients have been associated with prolonged mechanical ventilation, prolonged ICU and hospital lengths of stay, and higher mortality. ${ }^{(18)}$

For patients who do not require mechanical ventilation or vasopressors, general or disease-specific prognostic scores exist to risk stratify patients in ambulatory or emergency settings (e.g. qSOFA as a general score ${ }^{(3)}$ and CRB-65 score for pneumonia( $\left.{ }^{(19)}\right)$. Patients with a high risk of mortality should be transferred to either a high-dependency or ICU setting, depending on the local policy. The higher level of care frees up frontline staff and provides patients with more frequent monitoring, nursing care and physician attention.

\section{RECOMMENDATIONS FROM SEPSIS GUIDELINES}

The Surviving Sepsis Campaign guidelines provide comprehensive recommendations for sepsis management. ${ }^{(2)} \mathrm{A}$ key recommendation of these guidelines is timely completion of a clinical management bundle comprising blood culture, administration of broadspectrum antibiotics and fluid loading. Patients with sepsis should complete all bundle components within three hours of presentation, and preferably within one hour.

Frontline clinicians in the ambulatory or general ward settings may find it challenging to follow these guidelines strictly, given their limited access to tools such as point-of-care testing, urgent laboratory services, bedside imaging, invasive arterial pressure measurement and vasopressors. Such limitations also preclude a firm diagnosis of sepsis, necessitating alternative but pragmatic labels such as possible sepsis.

Nonetheless, frontline clinicians play a critical role in early identification and treatment of patients with sepsis. The key starting point is suspecting infection. Subsequently, possible sepsis needs to be rapidly identified via clinical gestalt, when capillary refill time exceeds three seconds or when the qSOFA score is $\geq 2$ (Fig. 1). Using the mnemonic 'SAFE', a bundle of interventions needs to be completed within three hours of presentation (preferably within one hour): sampling for cultures, antibiotic administration, fluid challenge and escalation (Box 2). Notice that a specific blood pressure level is not being targeted, as normal-looking blood pressure does not equate to normal perfusion, and low blood pressure unresponsive to fluid boluses should not provoke continued fluid administration.

\section{TAKE HOME MESSAGES}

1. The key to identification of sepsis is suspicion of infection, which requires clinical experience and skill.

2. Possible septic shock can be diagnosed when a patient has infection and any two of the following three criteria (qSOFA score): (a) altered mental status (i.e. Glasgow Coma Scale score $<15$ ); (b) respiratory rate $\geq 22$ breaths/min; and (c) systolic blood pressure $\leq 100 \mathrm{mmHg}$.

3. Possible sepsis can also be diagnosed when a patient has infection and hypoperfusion demonstrated by a capillary refill time $>3$ seconds or blood lactate $>2 \mathrm{mmol} / \mathrm{L}$.

4. Possible sepsis should be treated as rapidly as confirmed sepsis. 


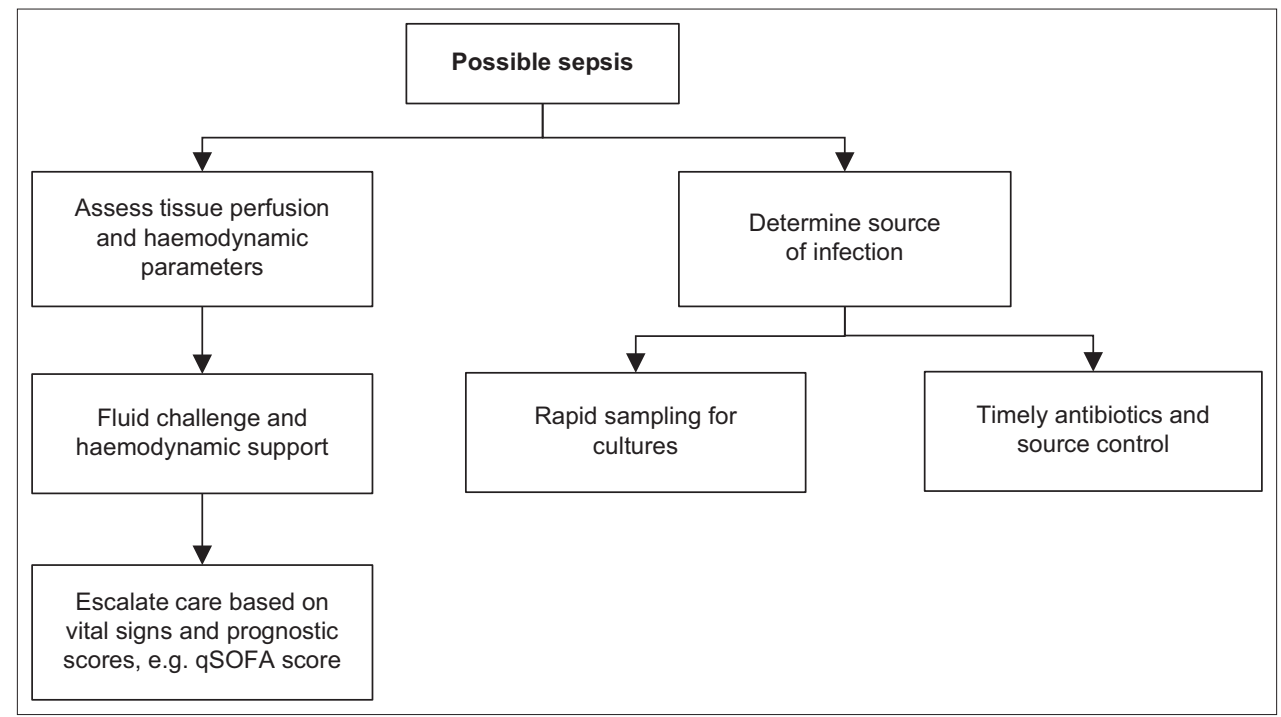

Fig. 1 Flowchart shows optimal sepsis management for the frontline clinician.

5. Timely treatment of sepsis includes: (a) administration of broad-spectrum intravenous antibiotics; (b) source control (e.g. drainage of pus); (c) fluid challenge with a fast intravenous bolus of normal saline or balanced crystalloid; and (d) escalation to an appropriate level of care.

You correctly recognised sepsis due to left pyelonephritis. The calculated qSOFA score was 2 out of 3 points, as evidenced by a respiratory rate of 22 breaths/min and a systolic blood pressure of $100 \mathrm{mmHg}$. Capillary refill time was delayed at four seconds, suggesting occult hypoperfusion, even in the absence of overt hypotension. As intravenous antibiotics were unavailable in the clinic, you called for an ambulance. While waiting, you inserted a 20-gauge intravenous cannula and administered $500 \mathrm{~mL}$ of normal saline over the next 30 minutes. The heart rate of the patient fell to 100 beats/min and blood pressure rose to $120 / 60 \mathrm{mmHg}$. The ambulance arrived soon after to take the patient to the hospital. A week later, a grateful patient returned to your clinic. She informed you that although she had Escherichia coli bacteriaemia from left pyelonephritis, she did not require intensive care and was discharged after spending five days in the general ward. This excellent outcome demonstrates the importance of timely prehospital initiation of sepsis care.

\section{REFERENCES}

1. Martins FS, Guedes GG, Santos TM, de Carvalho-Filho MA. Suspected infection in afebrile patients: Are they septic? Medicine (Baltimore) 2017; 96:e6299.

2. Evans L, Rhodes A, Alhazzani W, et al. Surviving sepsis campaign: international guidelines for management of sepsis and septic shock 2021. Intensive Care Med $2021 ; 47: 1181-247$
3. Seymour CW, Liu VX, Iwashyna TJ, et al. Assessment of clinical criteria for sepsis: for the third international consensus definitions for sepsis and septic shock (Sepsis-3). JAMA 2016; 315:762-74.

4. Rudd KE, Johnson SC, Agesa KM, et al. Global, regional, and national sepsis incidence and mortality, 1990-2017: analysis for the Global Burden of Disease Study. Lancet 2020; 395:200-11.

5. Kumar A, Roberts D, Wood KE, et al. Duration of hypotension before initiation of effective antimicrobial therapy is the critical determinant of survival in human septic shock. Crit Care Med 2006; 34:1589-96.

6. Hernández G, Ospina-Tascón GA, Damiani LP, et al. Effect of a resuscitation strategy targeting peripheral perfusion status vs serum lactate levels on 28day mortality among patients with septic shock: the ANDROMEDA-SHOCK randomized clinical trial. JAMA 2019; 321:654-64.

7. Garnacho-Montero J, Gutiérrez-Pizarraya A, Escoresca-Ortega A, FernandezDelgado E, Lopez-Sánchez JM. Adequate antibiotic therapy prior to ICU admission in patients with severe sepsis and septic shock reduces hospital mortality. Crit Care 2015; 19:302.

8. Vincent JL, Brealey D, Libert N, et al; Rapid Diagnosis of Infections in the Critically III Team. Rapid diagnosis of infection in the critically ill, a multicenter study of molecular detection in bloodstream infections, pneumonia, and sterile site infections. Crit Care Med 2015; 43:2283-91.

9. Li Y, Guo J, Yang H, et al. Comparison of culture-negative and culture-positive sepsis or septic shock: a systematic review and meta-analysis. Crit Care 2021; 25:167.

10. Martin GS, Mannino DM, Eaton S, Moss M. The epidemiology of sepsis in the United States from 1979 through 2000. N Engl J Med 2003; 348:1546-54.

11. Tan CW, Chlebicki MP. Urinary tract infections in adults. Singapore Med J 2016; 57:485-90

12. Bilbao-Meseguer I, Rodriguez-Gascón A, Barrasa H, Isla A, Solinís MÁ. Augmented renal clearance in critically ill patients: a systematic review. Clin Pharmacokinet 2018; 57:1107-21.

13. Martínez ML, Ferrer R, Torrents E, et al; Edusepsis Study Group. Impact of source control in patients with severe sepsis and septic shock. Crit Care Med 2017; 45:11-9.

14. Messina A, Palandri C, De Rosa S, et al. Pharmacodynamic analysis of a fluid challenge with $4 \mathrm{ml} \mathrm{kg}^{-1}$ over 10 or 20 min: a multicenter cross-over randomized clinical trial. J Clin Monit Comput 2021 Sep 8; 1-11. [Epub ahead of print]

15. See KC, Mukhopadhyay A, Lau SC, et al. Shock in the first $24 \mathrm{~h}$ of intensive care unit stay: observational study of protocol-based fluid management. Shock 2015; 43:456-62.

16. Zampieri FG, Machado FR, Biondi RS, et al; BaSICS investigators and the BRICNet members. Effect of intravenous fluid treatment with a balanced solution vs $0.9 \%$ saline solution on mortality in critically ill patients: the BaSICS randomized clinical trial. JAMA 2021; 326:1-12. [Epub ahead of print]

17. Aya HD, Ster IC, Fletcher N, et al. Pharmacodynamic analysis of a fluid challenge. Crit Care Med 2016; 44:880-91.

18. Mohr NM, Wessman BT, Bassin B, et al. Boarding of critically ill patients in the emergency department. Crit Care Med 2020; 48:1180-7.

19. Bauer TT, Ewig S, Marre R, Suttorp N, Welte T; CAPNETZ Study Group. CRB65 predicts death from community-acquired pneumonia. J Intern Med 2006; 260:93-101. 


\section{SINGAPORE MEDICAL COUNCIL CATEGORY 3B CME PROGRAMME} (Code SMJ 202201A)

1. Infection can be ruled out if a young, immunocompetent patient is afebrile.

2. Sepsis is a combination of infection and fever.

3. Septic shock is a combination of sepsis and hypotension.

4. The mean arterial pressure is the sum of one-third of the systolic blood pressure and two-thirds of the diastolic blood pressure.

5. Hypotension is defined as a mean arterial pressure $<65 \mathrm{mmHg}$.

6. Hypoperfusion can exist when mean arterial pressure is $70 \mathrm{mmHg}$.

7. One criterion of the qSOFA (quick Sequential Organ Failure Assessment) score is a respiratory rate $\geq 22$ breaths/min.

8. The qSOFA score is more sensitive than specific for diagnosing sepsis.

9. A capillary refill time of three seconds is abnormal.

10. An elevated blood lactate level of $4 \mathrm{mmol} / \mathrm{L}$ can exist without tissue hypoperfusion.

11. For patients with sepsis, antibiotics may be given within six hours of presentation.

12. Culture-negative sepsis and culture-positive sepsis have similar mortality.

13. Full doses of intravenous antibiotics can be administered initially for septic patients with renal impairment.

14. Surgery for source control in septic patients should not be delayed if hypoperfusion and hypotension cannot be fully reversed using medications alone.

15. A positive haemodynamic response to fluid therapy involves an increase of cardiac output or stroke volume of at least $10 \%$.

16. The initial choice of fluid for fluid challenge can be intravenous hydroxyethyl starch.

17. Antibiotics should not be given within one hour of sepsis presentation, as this risks antibiotic overuse and adverse drug reactions.

18. Blood cultures must be done as rapidly as possible to avoid delay of antibiotic administration in sepsis/septic shock.

19. Fluid challenge need not be considered if the mean arterial pressure is $70 \mathrm{mmHg}$.

20. Fluid challenge may not be needed if the capillary refill time is one second.

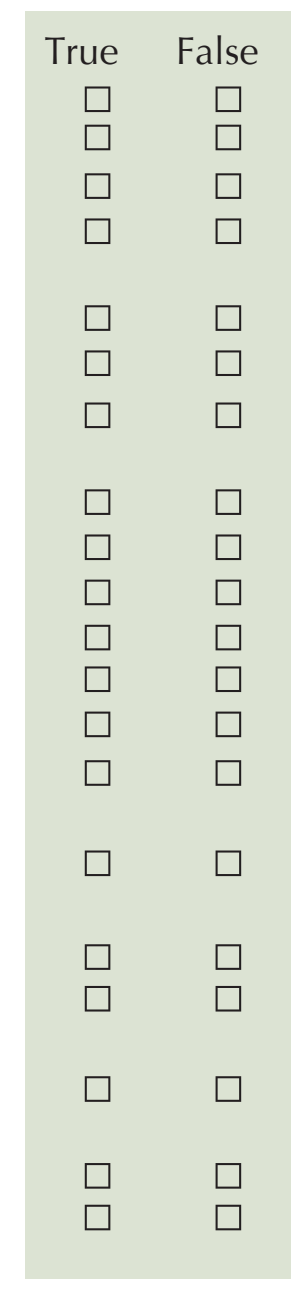

\section{Doctor's particulars:}

Name in full:

MCR no.:

Specialty:

Email:

\section{SUBMISSION INSTRUCTIONS:}

Visit the SMJ website: http://www.smj.org.sg/current-issue and select the appropriate quiz. You will be redirected to the SMA login page.

For SMA member: (1) Log in with your username and password (if you do not know your password, please click on 'Forgot your password?'). (2) Select your answers for each quiz and click 'Submit'.

For non-SMA member: (1) Create an SMJ CME account or log in with your SMJ CME username and password (for returning users). (2) Make payment of SGD 21.40 (inclusive of $7 \%$ GST) via PayPal to access this month's quizzes. (3) Select your answers for each quiz and click 'Submit'.

RESULTS:

(1) Answers will be published online in the SMJ March 2022 issue. (2) The MCR numbers of successful candidates will be posted online at the SMJ website by 31 March 2022 (3) Passing mark is $60 \%$. No mark will be deducted for incorrect answers. (4) The SMJ editorial office will submit the list of successful candidates to the Singapore Medical Council. (5) One CME point is awarded for successful candidates. (6) SMC credits CME points according to the month of publication of the CME article (i.e. points awarded for a quiz published in the December 2021 issue will be credited for the month of December 2021, even if the deadline is in February 2022).

Deadline for submission (January 2022 SMJ 3B CME programme): 12 noon, 24 March 2022. 